\title{
Origin of the hysteresis in bilayer 2D systems in the quantum Hall regime
}

\author{
L.H. Ho, ${ }^{1,2, \text {, }}$ L.J. Taskinen, ${ }^{1}$ A.P. Micolich, ${ }^{1, \text { 由 }}$ A.R. Hamilton, ${ }^{1}$ P. Atkinson, ${ }^{3}$ and D.A. Ritchie ${ }^{3}$ \\ ${ }^{1}$ School of Physics, University of New South Wales, Sydney NSW 2052, Australia \\ ${ }^{2}$ CSIRO Materials Science and Engineering, P.O. Box 218, Lindfield NSW 2070, Australia \\ ${ }^{3}$ Cavendish Laboratory, University of Cambridge, Cambridge CB3 OHE, United Kingdom
}

(Dated: December 6, 2018)

\begin{abstract}
The hysteresis observed in the magnetoresistance of bilayer 2D systems in the quantum Hall regime is generally attributed to the long time constant for charge transfer between the $2 \mathrm{D}$ systems due to the very low conductivity of the quantum Hall bulk states. We report electrometry measurements of a bilayer $2 \mathrm{D}$ system that demonstrate that the hysteresis is instead due to non-equilibrium induced current. This finding is consistent with magnetometry and electrometry measurements of single $2 \mathrm{D}$ systems, and has important ramifications for understanding hysteresis in bilayer 2D systems.
\end{abstract}

PACS numbers: 07.50.-e,73.43.-f,75.60.Ej

The study of two-dimensional electron systems (2DESs) has led to much interesting physics including the Nobel prize winning discoveries of the integer ${ }^{\underline{1}}$ and fractional ${ }^{2}$ quantum Hall effects. These effects are dramatic examples of the vital role that dimensionality, quantization and electron-electron interactions play in the physics of semiconductor devices. Deeper insight into the role of interactions can be obtained by locating a second 2DES in close proximity. These 'bilayer' $2 \mathrm{D}$ electron systems have received significant interest as routes to realizing exotic new electronic ground states ranging from excitonic Bose-Einstein condensates ${ }^{3}$ through to quantum Hall ferromagnets. 4

The latter has been a particular focus for attention with numerous reports of hysteresis - a common hallmark of ferromagnetic behavior - in bilayer 2D systems in the quantum Hall regime ${ }^{5-10}$ Bilayer $2 \mathrm{D}$ systems provide an additional layer of complexity to studies of magnetic ordering because in addition to the electron spin, these systems have a 'pseudospin' degree of freedom corresponding to which of the two layers an electron occupies. For example, Piazza et al. studied a wide quantum well containing two 2DESs, and showed that a first-order magnetic phase transition could be induced by careful tuning of the energetic alignment between a spin-up Landau level in one 2DES and a spin-down Landau level in the other, an effect requiring both spin and pseudospin to explain $\underline{\underline{5}}$

Subsequent papers have reported more widespread hysteresis. Zhu et al. measured the longitudinal magnetoresistance $R_{x x}$ of a 2DES with a nearby, parallel impurity channel and observed resistance spikes coinciding with a number of the integer quantum Hall minima, and hysteresis between data obtained with increasing/decreasing magnetic field at the edges of these minima. ${ }^{7}$ Similar behavior was reported by Tutuc et al. $\stackrel{8}{\underline{n}}$ and Misra et al $\underline{10}$ in bilayer 2D hole systems and by Pan et $a l^{\underline{\underline{9}}}$ in a bilayer 2DES. In each case, the authors explained these effects as being entirely due to impeded charge transfer between the two 2D systems, which occurs in the following way. At integer filling factor, each 2DES will undergo a sudden change in its chemical poten- tial due to Landau level population/depopulation. This leads to a non-equilibrium imbalance in the chemical potentials of the two 2D systems, which is overcome by charge migration via the ohmic contacts connecting the two $2 \mathrm{D}$ systems. However, this charge migration is impeded by the localization of the bulk states in the quantum Hall regime, leading to a large $R C$ time-constant, and hence the observed hysteresis ${ }^{?}$

The problem with this charge transfer mechanism is that it ignores another well established cause of hysteresis in 2D systems in the quantum Hall regime non-equilibrium induced currents. Large and long-lived induced currents can circulate within the dissipationless edge states associated with the quantum Hall effect. These currents can be driven for instance by the induced emf caused by a changing magnetic field. They produce a hysteretic magnetization signal that has been widely observed in magnetometry studies of single layer 2D systems, 11 both in response to a changing magnetic field $B, \frac{12}{12}$ and changing density in the $2 \mathrm{D}$ system at constant $B \stackrel{13}{n}$ These induced currents also generate a large hysteretic electrostatic potential (of the order $\sim 10 \mathrm{mV}$ ) that has been observed in single-electron transistor electrometry studies of single $2 \mathrm{D}$ systems $\stackrel{14}{\underline{4}}$ Thus it would be surprising if non-equilibrium induced current did not play some role, perhaps even a dominant one, in the hysteresis reported in bilayer $2 \mathrm{D}$ systems $\underline{\underline{7} \underline{\underline{10}}}$

It is perhaps understandable that non-equilibrium induced current in bilayer 2D systems has received limited atttention to this point, because preceding studies have relied solely on traditional transport measurements, which cannot provide any direct experimental evidence for the relative contributions of the two mechanisms to the hysteresis. In this paper, we present electrometry measurements of a bilayer 2D system obtained using a method recently developed by Ho et al. $\frac{15}{15}$ and obtain a clear experimental signature for the dominance of non-equilibrium induced current over charge transfer (see Figs. 1(b/c) and 2). Our data clearly demonstrates that charge transfer alone cannot be responsible for the hysteresis effects previously reported in bilayer 2D systems,,-10 and is at best a very small contribution towards 

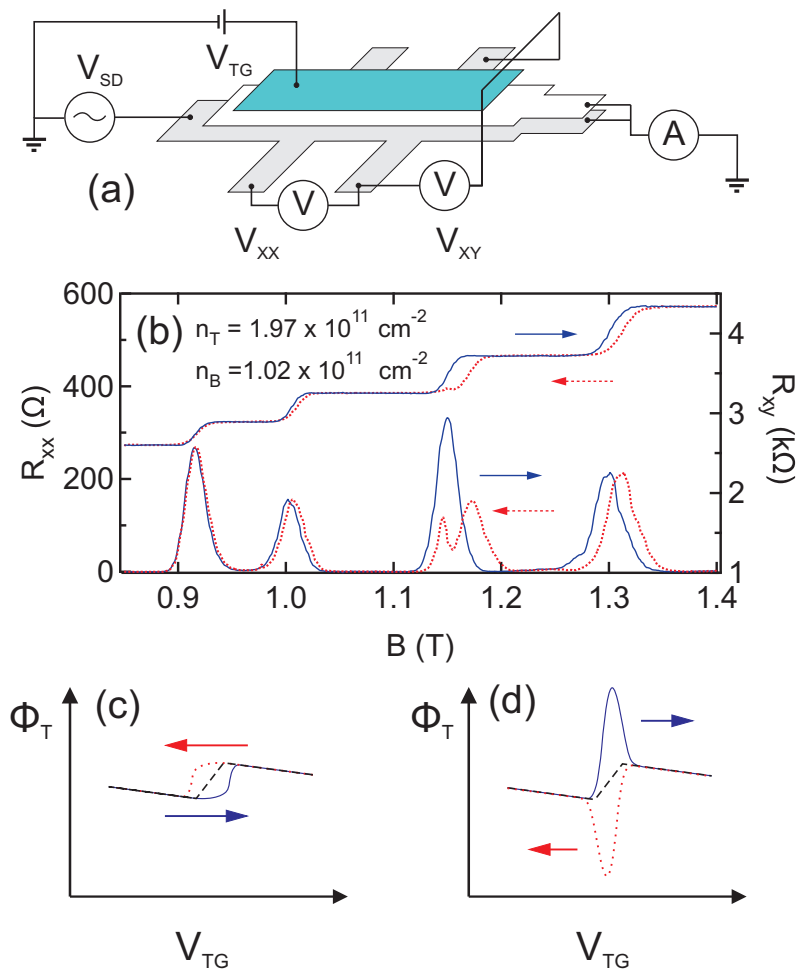

FIG. 1:

(color online) (a) A schematic of the device and measurement circuit with (from bottom) the lower 2DES in grey, the upper 2DES in white and the top-gate in blue. The upper 2DES shares only one common contact with the lower

2DES, which is connected to ground, allowing the upper

2DES density to respond to changes in top gate bias. The measured Hall voltage $V_{x y}$ can be used to obtain the upper 2DES chemical potential $\mu_{T}$. (b) The measured longitudinal resistance $R_{x x}$ (lower two traces) and Hall resistance $R_{x y}$ (upper two traces) in the lower 2DES versus magnetic field

$B$ demonstrating similar hysteresis to that previously reported in bilayer $2 \mathrm{D}$ systems ${ }^{7,9,10}(\mathrm{c})$ and $(\mathrm{d})$ are schematics illustrating the expected behavior of the measured electrostatic potential $\phi_{T}$ in the upper 2DES as a

function of top-gate bias $V_{T G}$ for the charge transfer and non-equilibrium induced current mechanisms respectively. In

(b)-(d) the blue solid/red dotted lines indicate data taken with increasing/decreasing $B$ or $V_{T G}$.

this effect.

Our device was produced using a double quantum well heterostructure featuring two $20 \mathrm{~nm}$ wide GaAs quantum wells separated by a $30 \mathrm{~nm} \mathrm{Al}_{0.33} \mathrm{Ga}_{0.67} \mathrm{As}$ barrier, giving an effective 2DES separation of $d=50 \mathrm{~nm}$. Standard semiconductor processing techniques were used to produce a Hall-bar with NiGeAu ohmic contacts that penetrate both quantum wells. The connections between the upper 2DES and all but one of the ohmic contacts are severed using a set of negatively biased 'depletion' gates $\frac{15}{}$ This isolates the upper 2DES from the measurement circuit, aside from a connection to ground via the drain contact that enables the upper 2DES density to be tuned by applying a voltage $V_{T G}$ to the top-gate, as per the schematic in Fig. 1(a). With the top-gate unbiased, the upper (lower) 2DES has a mobility of $1.2 \times 10^{6} \mathrm{~cm}^{2} / \mathrm{Vs}$ $\left(1.4 \times 10^{6} \mathrm{~cm}^{2} / \mathrm{Vs}\right)$ and density $n_{\mathrm{T}}=2.00 \times 10^{11} \mathrm{~cm}^{-2}$ $\left(n_{\mathrm{B}}=1.98 \times 10^{11} \mathrm{~cm}^{-2}\right)$. All electrical measurements were performed at a temperature $\sim 50 \mathrm{mK}$ using fourterminal lock-in techniques with an excitation voltage of $100 \mu \mathrm{V}$ at $17 \mathrm{~Hz}$.

We commence by demonstrating that our device shows hysteresis similar to that previously observed in bilayer 2 D systems ${ }^{7-10}$ In Fig. 1(b), we plot the longitudinal and Hall resistances $R_{x x}$ and $R_{x y}$ of the lower 2DES measured with increasing (blue solid line) and decreasing (red dotted line) perpendicular magnetic field $B$. To compare directly with previous work, we deliberately imbalance the two 2DESs (i.e., $n_{T} \neq n_{B}$ ) when making this measurement. We observe clear hysteresis in regions where $R_{x x} \neq 0$, consistent with the literature, for example, cf. Fig. 1 of Zhu et al.,$^{\frac{7}{2}}$ Fig. 2 of Pan et al.,$\frac{9}{\underline{y}}$ or Fig. 4 of Misra et al. ${ }^{10}$

We now turn to measurements of the electrostatic potential $\phi_{T}$ in the upper 2DES. The lower 'sensor' 2DES is used as a capacitively-coupled electrometer $\frac{15}{2}$ whereby changes in $\phi_{T}$ lead to changes in the lower 2DES density $\Delta n_{B}=\epsilon \Delta \phi_{\mathrm{T}} /(e d)$. The latter is observed by monitoring $R_{x y}$ as the upper 2 DES density $n_{\mathrm{T}}$ is varied by sweeping $V_{T G}$ between $0 \mathrm{~V}$ and the depletion of the upper 2DES at $V_{T G}=-0.3 \mathrm{~V}$. For maximum sensitivity, we choose a fixed 'operating point' in magnetic field $B$ where the lower 2DES is at the midpoint between two Hall plateaus, and the relationship between $R_{x y}$ and $n_{\mathrm{B}}$ is obtained by characterizing the shape of this quantum Hall transition 15

Changes in $\phi_{T}$ result from changes in the upper 2DES chemical potential $\mu_{T}$, and the electrostatic potential $\phi_{N E C}$ due to non-equilibrium induced current flowing in the upper 2DES such that $\Delta \phi_{T}=\Delta \mu_{T} / e+\phi_{N E C}$. Before looking at the actual data, we first consider the expected behavior of $\phi_{T}$ under the two possible mechanisms for the hysteresis at fixed $B$. At complete equilibrium (i.e., there is no non-equilibrium induced current $\phi_{N E C}=0$ or charge transfer hysteresis), $\mu_{T}$ would follow a sawtoothshaped path (black dashed line in Fig. 1(c/d)) with increasing $n_{T}$ or $V_{T G}$, falling gradually at fixed Landau level occupation due to negative compressibility, and rising rapidly at integer filling factor $\nu_{T}$ due to repopulation of the lowest unoccupied Landau level ${ }^{15}$ In the charge transfer mechanism, the long $R C$ time-constant should lead to a hysteretic $\mu_{T}$ resulting in the behavior shown in the blue solid and red dashed lines in Fig. 1(c). The most significant features are that $\mu_{T}$ evolves monotonically between the potentials immediately before and after the transition, and always lags the equilibrium transition point by an amount directly proportional to the sweep rate. The expected behavior for the non-equilibrium current mechanism is shown in Fig. 1(d). Here $\phi_{T}$ leads the equilibrium transition point, and significantly overshoots the potential on either side due to $\phi_{N E C} \stackrel{14}{=}$ In practice, this overshoot is large, the hysteresis is an order of mag- 


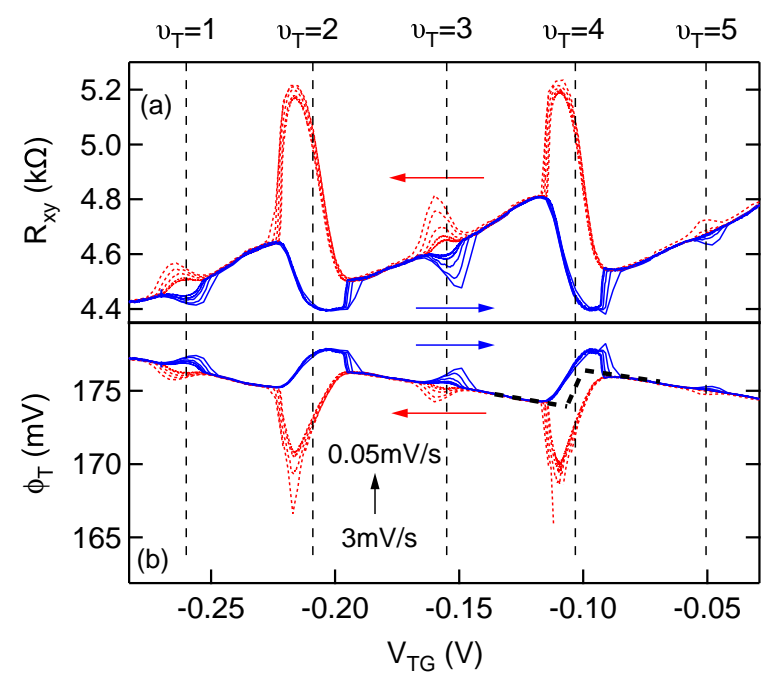

FIG. 2:

(color online) (a) The measured lower 2DES Hall resistance $R_{x y}$ and (b) the corresponding upper 2DES electrostatic potential $\phi_{T}$ versus top-gate voltage $V_{T G}$ for decreasing $V_{T G}$ (red dotted lines) and increasing $V_{T G}$ (blue solid lines). The dashed black line in (b) highlights the ideal behavior of $\mu_{T}$ in the absence of any hysteresis. In each case, five traces are presented, obtained at $V_{T G}$ sweep rates of 3, 1, 0.3, 0.1 and $0.05 \mathrm{mV} / \mathrm{s}$, with the largest deviation from ideal behavior occurring at highest sweep-rate.

nitude larger than the equilibrium change in $\mu_{T}$.

In Fig. 2(a) and (b) we show the measured $R_{x y}$ and corresponding $\phi_{T}$ versus $V_{T G}$ at a variety of sweep rates between 0.05 and $3 \mathrm{mV} / \mathrm{s}$. The up-sweeps towards increased $V_{T G}$ are presented with blue solid lines and the down-sweeps are presented with red dashed lines. Hysteresis is clearly evident at integer filling factor $\nu_{T}$ in Fig. 2, with the equilibrium change in chemical potential highlighted by the dashed black line near $\nu_{T}=4$ in Fig. 2(b). The overshoot observed undeniably points towards non-equilibrium induced current being generated in the upper 2DES $\underline{13}$ This overshoot is also visible at odd $\nu_{T}$. Here the equilibrium changes in chemical potential are due to the Zeeman spin-splitting and are too small to be observed, however the hysteresis remains clearly visible. Another notable feature is that the hysteresis is significantly stronger at even $\nu_{T}$ than at odd $\nu_{T}$. This is consistent with the non-equilibrium current mechanism, since the larger change in $\mu_{T}$ that occurs at even $\nu_{T}$ will produce more dissipationless edge states in the upper 2DES, which result in larger induced currents and correspondingly stronger hysteresis. The same behavior is widely observed in magnetometry $\stackrel{11}{1}$ and electrometry 14 measurements of single $2 \mathrm{D}$ systems.

To delve further into the data in Fig. 2, we must first consider how the hysteresis should depend on rate of change of $V_{T G}$ in the two mechanisms. To first order, charge transfer should give no sweep rate dependence because the factor limiting charge migration from one
2DES to the other is the very low conductivity of the bulk states, which is independent of the rate of change in $V_{T G}$. However, since the sweep rate also affects the rate at which the equilibrium chemical potential in each 2DES changes, there will be a weak sweep rate dependence in the charge transfer mechanism. In contrast, the sweep rate dependence for the non-equilibrium current mechanism should be strong. For the case where $B$ is swept rather than $V_{T G}$, the explanation is quite simple - the non-equilibrium induced current in the edge states is just the eddy current, as described by Faraday's law $\epsilon \propto-d B / d t$, where $\epsilon$ is the induced electromotive force (emf). If we sweep $V_{T G}$ instead, then the change in density results in charge flowing into/out of the $2 \mathrm{DES}$ via the ohmic contacts. This flow is initially directed towards the centre of the 2DES, but is rapidly channeled into the edge states by the Lorentz force. We note that the sweep rate dependence of real induced current will differ from the simple pictures discussed above due to the breakdown of the quantum Hall effect 11 and also a capacitive mechanism in gated samples $\frac{16}{\underline{1}}$

With the expected behavior in mind, at first sight, the measured sweep rate dependence in Fig. 2(b) appears to be inconsistent with the dominance of either mechanism - the sweep rate dependence at odd $\nu_{T}$ is strong but for even $\nu_{T}$ it appears to be very weak. For example, in Fig. 2(a) the peak-peak amplitude of the hysteresis 'loop' at $\nu_{T}=3$ varies from $53 \Omega$ at a sweep rate $d V_{T G} / d t=0.05 \mathrm{mV} / \mathrm{s}$ to $334 \Omega$ at $d V_{T G} / d t=3 \mathrm{mV} / \mathrm{s}$, an increase of over $500 \%$ at $\nu_{T}=3$. In comparison, for $\nu_{T}=4$ the amplitude increases from $786 \Omega$ to $857 \Omega$ for the same sweep rate change, the corresponding difference being only $9 \%$. This lack of sweep rate dependence at even $\nu_{T}$ is ultimately due to a limitation in our electrometry technique. As mentioned earlier, we sense changes in the electrostatic potential in the upper 2DES by setting a magnetic field 'operating point' such that the lower 2DES is directly in between two Hall plateaus (i.e., to half integer filling factor $\nu_{B}$ in the lower 2DES) and monitoring the lower 2DES Hall resistance $R_{x y} .15$ While this gives us optimum sensitivity, since $d R_{x y} / d \phi_{T}$ is a maximum at half integer $\nu_{B}$, the range is limited because as $R_{x y}$ approaches the adjacent Hall plateaus this sensitivity drops to zero. The data in Fig. 2 was obtained at $B=1.55 \mathrm{~T}$ (i.e., $\nu_{B}=5.5$ ), which means that the lower 2DES 'sensor' sensitivity is maximal at $R_{x y}=4693 \mathrm{k} \Omega$ and diminishes monotonically to zero by $R_{x y}=4302$ and $5162 \mathrm{k} \Omega$. A careful look at the abscissa in Fig. 2(a) reveals that the hysteresis loops at even $\nu_{T}$ have reached these zero sensitivity limits, and thus the lack of sweep-rate dependence in $R_{x y}$ does not imply a lack of sweep rate dependence in the size of the induced current.

Overcoming this sensitivity problem is not straightforward. The most obvious solution would be to shift the operating point to obtain a reduced half-integer $\nu_{B}$ where the $R_{x y}$ range between the adjacent Hall plateaus is greater. Unfortunately, the higher magnetic field re- 

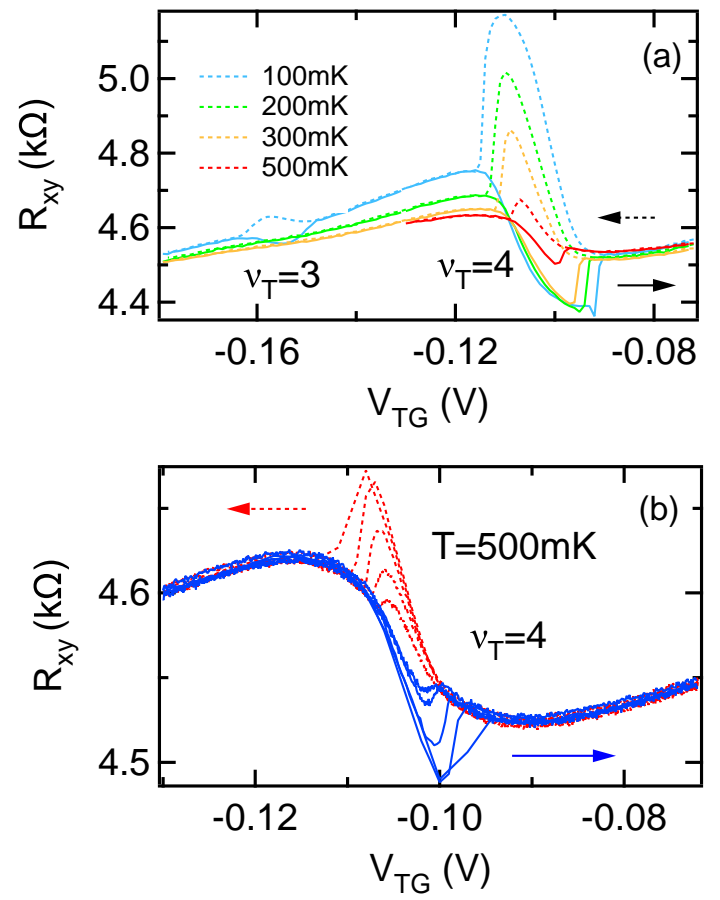

FIG. 3:

The Hall resistance $R_{x y}$ vs $V_{T G}$ obtained at $B=1.55 \mathrm{~T}$ for (a) a fixed sweep rate of $1 \mathrm{mV} / \mathrm{s}$ for temperatures $T$ of 100 , 200, 300 and $500 \mathrm{mK}$, and (b) a fixed temperature $T=500 \mathrm{mK}$ for sweep rates of $0.05,0.1,0.3,1$ and $3 \mathrm{mV} / \mathrm{s}$. In both panels, up-sweeps/down-sweeps are indicated with solid/dashed lines.

quired to achieve this also reduces $\nu_{T}$, pushing the data in Fig. 2(a) outside the available range of $V_{T G}$. A better solution would be to introduce a back-gate in the device so that the lower 2DES density can be tuned independently of the upper 2DES density. Then it would be possible to implement a feedback mechanism, whereby the lower 2DES is maintained at the optimum half-integer filling factor with the magnetic field fixed, with the back-gate voltage required to do used as the measurable quantity in detecting changes in $\phi_{T}$. This will not be simple to implement, and is the goal of future work; in this paper, we will follow another avenue to demonstrate that there is in fact a strong sweep rate dependence at even $\nu_{T}$ con- sistent with non-equilibrium induced current being the dominant contribution to the hysteresis.

In Fig. 3(a), we present the temperature dependence of the hysteresis loops at $\nu_{T}=3$ and 4 for a fixed sweep rate of $1 \mathrm{mV} / \mathrm{s}$. As the temperature $T$ is increased towards $500 \mathrm{mK}$, the hysteresis loops at both odd and even $\nu_{T}$ diminish in amplitude. This is also observed in magnetization measurements of a single 2DES in the quantum Hall regime, ${ }^{16}$ and is due to increasing dissipation in the edge states with temperature 17,18 However, this also carries a positive side-effect, which is a small increase in the dynamic range of the lower $2 \mathrm{DES}$ as a sensor for the upper 2DES electrostatic potential. The hysteresis at $\nu_{T}=3$ is quenched by $T=200 \mathrm{mK}$, and while the hysteresis at $\nu_{T}=4$ remains at $T=500 \mathrm{mK}$, it is sufficiently diminished that it stays well within the sensitivity range of the upper $2 \mathrm{DES}$. Thus we repeat the sweep rate dependence study for $\nu_{T}=4$ at $T=500 \mathrm{mK}$, the results are presented in Fig. 3(b). Here the strong sweep rate dependence expected for the non-equilibrium induced current is indeed observed, with the peak-to-peak amplitude increasing by $243 \%$ as the sweep rate is increased from 0.05 to $3 \mathrm{mV} / \mathrm{s}$. This confirms that the lack of sweep rate dependence for even $\nu_{T}$ in Fig. 2 was due to the large induced current driving the sensor 2DES into insensitive regions.

In conclusion, we have studied the origin of the hysteresis observed in bilayer $2 \mathrm{D}$ systems in the quantum Hall regime using a newly developed electrometry technique ${ }^{15}$ that allows direct measurements of the electrostatic potential in one of the two $2 \mathrm{D}$ systems. We observe hysteresis 'loops' that point very strongly to the dominance of non-equilibrium induced current over impeded charge transfer as a cause of the hysteresis recently reported in bilayer $2 \mathrm{D}$ systems $?^{7-10}$ Our findings are consistent with previous measurements of hysteresis in single $2 \mathrm{D}$ systems 11,14

This work was funded by Australian Research Council (ARC). L.H.H. acknowledges financial support from the UNSW and the CSIRO. ARH and APM acknowledge financial support from ARC Professorial and Future Fellowships, respectively. The authors thank Dr Jack Cochrane for technical support. This work was performed in part using the NSW node of the Australian National Fabrication Facility (ANFF).
* Electronic address: laphang@phys.unsw.edu.au

† Electronic address: adam.micolich@gmail.com

1 K.v. Klitzing, G. Dorda and M. Pepper, Phys. Rev. Lett. 45, 494 (1980).

2 D.C. Tsui, H.L. Störmer and A.C. Gossard, Phys. Rev. Lett. 48, 1559 (1982).

3 J.P. Eisenstein and A.H. MacDonald, Nature 432, 691 (2004).

4 S.M. Girvin, Physics Today 53(6), 39 (2000).
${ }^{5}$ V. Piazza, V. Pellegrini, F. Beltram, W. Wegscheider, T. Jungwirth and A.H. MacDonald, Nature 402, 638 (1999).

6 E.P. De Poortere, E. Tutuc, S.J. Papadakis and M. Shayegan, Science 290, 1546 (2000).

7 J. Zhu, H.L. Stormer, L.N. Pfeiffer, K.W. Baldwin, and K.W. West, Phys. Rev. B 61, R13361 (2000).

8 E. Tutuc, R. Pillarisetty, S. Melinte, E.P. De Poortere, and M. Shayegan, Phys. Rev. B 68, 201308(R) (2003).

9 W. Pan, J.L. Reno, and J.A. Simmons, Phys. Rev. B 71, 
153307 (2005).

10 S. Misra, N.C. Bishop, E. Tutuc, and M. Shayegan, Phys. Rev. B 78, 035322 (2008).

11 A. Usher and M. Elliott, J. Phys.: Condens. Matter 21, 103202 (2009).

12 J.P. Watts, A. Usher, A.J. Matthews, M. Zhu, M. Elliott, W.G. Herrenden-Harker, P.R. Morris, M.Y. Simmons, and D.A. Ritchie, Phys. Rev. Lett. 81, 4220 (1998).

13 D.R. Faulhaber and H.W. Jiang, Phys. Rev. B 72, 233308 (2005).

14 J. Huels, J. Weis, J. Smet, K.v. Klitzing and Z.R. Wasilewski, Phys. Rev. B 69, 085319 (2004).
15 L.H. Ho, L.J. Taskinen, A.P. Micolich, A.R. Hamilton, P. Atkinson, M. Pepper and D.A. Ritchie, Appl. Phys. Lett. 96, 212102 (2010).

16 N. Ruhe, G. Stracke, Ch. Heyn, D. Heitmann, H. Hardtdegen, Th. Schäpers, B. Rupprecht, M.A. Wilde and D. Grundler, Phys. Rev. B 80, 115336 (2009).

17 D.C. Tsui, H.L. Störmer and A.C. Gossard, Phys. Rev. B 25, 1405 (1982).

18 M.E. Cage, B.F. Field, R.F. Dziuba, S.M. Girvin, A.C. Gossard and D.C. Tsui, Phys. Rev. B 30, 2286 (1984). 